\title{
Early Processing of Threat Cues in Posttraumatic Stress Disorder-Evidence for a Cortical Vigilance-Avoidance Reaction
}

\author{
Hannah Adenauer, Steivan Pinösch, Claudia Catani, Hannah Gola, Julian Keil, Johanna Kißler, and \\ Frank Neuner
}

Background: The present study investigated the influence of posttraumatic stress disorder (PTSD) on early visual processing of affective stimuli in survivors of war and torture.

Methods: Trauma-exposed refugees with $(n=36)$ and without $(n=21)$ PTSD as well as unexposed control subjects $(n=16)$ participated in a magnetoencephalography study with pictures that varied in emotional content.

Results: We found evidence for a biphasic cortical response in patients with PTSD in comparison with the two control groups. In response to aversive (relative to neutral or positive) pictures, PTSD patients showed elevated cortical activity over right prefrontal areas as early as $130-160 \mathrm{msec}$ after stimulus onset followed by a decrease of the affect-related response in the parieto-occipital cortex at 206-256 msec.

Conclusions: The increased early activity in the right prefrontal cortex most likely represents an enhanced alarm response or the fear network toward aversive stimuli in PTSD, whereas the subsequent decreased activation in right parieto-occipital areas in response to aversive pictures seems to reflect the tendency to disengage from emotional content. This finding is consistent with the hypothesis of a vigilance-avoidance reaction pattern to threat in anxiety disorders and helps to reconcile contradicting results of over- and underresponsiveness in the sensory processing of threatening stimuli in PTSD.

Key Words: Attentional bias, neurophysiological correlates, processing of threatening stimuli, PTSD, traumatic stress, vigilance-avoidance

urrent theories of posttraumatic stress disorder (PTSD) consider alterations in the processing of threat cues as a core characteristic of PTSD (1). It is assumed that patients with PTSD show a cognitive bias toward unpleasant cues that indicate potential threat (e.g., observing violence or aggressive faces). This bias constitutes the physiological and emotional hyperresponsiveness of PTSD patients $(2,3)$ and is probably a reflection of alterations of basic fear-processing mechanisms. A distinct system (fear network) that enables the rapid detection of threat as well as the immediate initiation of a defensive reaction underlies the neuronal processing of fear. It involves subcortical structures including the amygdala $(4,5)$ as well as cortical regions, in particular the ventral prefrontal cortex $(6,7)$.

Several brain-imaging studies have confirmed that neuronal structures of fear-processing are overly reactive toward threat cues in PTSD. Recent literature reports hyperresponsivity of the amygdala (8) as well as the prefrontal cortex (7) toward aversive stimuli in PTSD subjects. In addition, studies measuring event-related brain potentials (ERPs) consistently found larger attention-related components (P3) after trauma stimuli (9)

Other studies, however, found the opposite effect, (i.e., a reduced cortical reactivity to threat cues in PTSD patients compared with nontraumatized control subjects) (10). Catani

From the Department of Psychology (HA, SP, HG, JKe, JKi), University of Konstanz, Konstanz, Germany; and the Department of Psychology (CC, FN), Bielefeld University, Bielefeld, Germany.

Address correspondence to Dr. Hannah Adenauer, Department of Psychology, University of Konstanz, 78457 Konstanz, Germany. E-mail: Hannah. Adenauer@uni-konstanz.de. et al. (11) as well as Weber et al. (12), for example, demonstrated that traumatized patients showed a significantly smaller affective modulation of occipital and parietal regions in response to aversive pictures.

One possible explanation for these conflicting findings might be the "vigilant-avoidant" pattern, which has been suggested to account for contradictory results from behavioral and eye-tracking attention studies with anxiety patients (13-15). According to this hypothesis, although aversive cues evoke a rapid response, anxious subjects subsequently initiate attentional avoidance as an attempt to alleviate the fear reaction $(13,15)$. It can be assumed that subjects with PTSD show a strong and immediate processing of aversive cues to allow a rapid detection of threat. This reaction might be essential for survival in a hostile environment with a high risk for retraumatization. Once a stimulus is categorized as threatening, however, further attention allocation toward the stimulus is not necessary and might even be obstructive for the initiation of a flight reaction.

In the present study, we investigated whether both opposing responses can be found in a single experiment: a hypervigilant cortical reaction followed by a subsequent avoidant response. For this purpose, we investigated the time course of the cortical reaction to aversive in comparison with neutral pictures in an event-related field (ERF) study with PTSD subjects. To allow a comparison with the results of recent electrophysiological studies with emotional pictures as stimuli, we adhered to the standard procedure of presenting three categories of images: pleasant, unpleasant, and neutral. In general, these studies found that the early posterior negativity component (EPN) (120-150 msec after stimulus onset) and the late positive potential (LPP) (past $300 \mathrm{msec}$ ) are modulated by the emotional quality of a stimulus, which indicates that motivationally relevant stimuli automatically direct attentional resources $(16,17)$

We expected that PTSD patients would show an increased neuronal excitation after aversive stimulation in the ventral 
prefrontal cortex, which plays a role in stimulus categorization and seems to be reactive to emotional stimuli $(6,18)$. After this early effect, we expected an attenuation of cortical processing in later time windows as a marker of attentional disengagement and cognitive avoidance in sensory processing areas in individuals with PTSD. To disentangle the impact of traumatic exposure and the influence of PTSD, three groups of participants were included in the experiment: PTSD subjects, Trauma Control subjects who reported a history of trauma exposure but did not fulfill PTSD criteria, and Unexposed control subjects. All subjects were refugees, asylum seekers, and immigrants.

\section{Methods}

\section{Participants}

A total of 73 immigrants from various crisis-affected countries participated in the study. Subjects included asylum seekers and refugees with a history of war and torture who came for treatment or expert opinion to the University of Konstanz Research and Outpatient Clinic for Refugees. In addition, healthy comparison participants with a similar ethnic background were recruited by announcements on campus bulletin boards.

Subjects were divided into three subgroups according to their clinical diagnoses and their traumatic life experiences: 36 participants with a diagnosis of PTSD according to DSM-IV (PTSD group), 21 subjects with a similar background but without current PTSD diagnosis (Trauma Control group), and 16 immigrants with no prior war and torture experiences (Unexposed Control group). Subjects with a current or past history of psychotic disorder or a current alcohol and substance dependence were excluded from the study. The present study is based to a large extent on the sample described in the study by Catani et al. (11). Because of the exclusion of a few subjects due to bad magnetoencephalography (MEG) data quality, there are minor differences with respect to sample sizes.

All participants underwent an extensive standardized clinical interview administered by experienced clinical psychologists and trained translators. The number of trauma experiences was assessed by the Life Events Checklist of the Clinician Administered P'TSD Scale; Clinical Administered PTSD Scale (CAPS) (19); and the vivo Checklist of War, Detention, and Torture events (20). The CAPS was used for the diagnosis of PTSD and the rating of PTSD symptoms. Furthermore, we assessed diagnoses of comorbid Axis I disorders with the Mini International Neuropsychiatric Interview (MINI) (21). The Hamilton Depression Rating Scale (HDRS) (22) was used to assess severity of depressive symptoms. Descriptive data as well as significant group differences in demographic and clinical variables are presented in Table 1.

\section{Stimuli and Presentation Procedure}

A total of 75 colored pictures were chosen from the International Affective Pictures System (23). The pictures were divided according to hedonic valence and emotional arousal: 25 aversive (e.g., mutilations, assaults, weapons), 25 pleasant (e.g., sports, happy couples, children), and 25 neutral pictures (e.g., neutral faces, household objects, landscapes) (specific images are listed in the supplement). These three categories differed significantly in terms of their normative valence ratings (pleasant: $7.4 \pm 1.6$, neutral: $4.9 \pm 1.3$, aversive: $2.4 \pm$
1.5). Although normative arousal ratings did not differ for pleasant and aversive contents, mean arousal levels for both emotional categories were significantly higher than pictures of neutral content (pleasant: $5.6 \pm 2.3$, neutral: $2.9 \pm 1.9$ ), aversive: $5.8 \pm 2.3$ ). Color spectra, contrast, and brightness of the pictures were matched across all three categories. Pictures were presented in a pseudorandom order with a video projector (JVC, DLA-G11E) on a gray plastic screen that was attached to the ceiling of the MEG chamber.

Because the design of the study included the analysis of the steady state signal evoked by the emotional stimuli (11), the pictures were presented in a flickering mode of $10 \mathrm{~Hz}$ for $4 \mathrm{sec}$. During the interstimulus interval that varied randomly between 6 and $8 \mathrm{sec}$, a black fixation cross was presented.

\section{Procedure}

Clinical interviews with trauma-exposed participants were carried out 1 week before MEG recording to prevent emotional priming of the reactions to the stimuli by the diagnostic interview. Upon arrival at the laboratory, the participants were provided with a verbal and written explanation of the procedure and gave informed consent to participate. Subjects were seated in a magnetically shielded chamber, and their head shapes were digitized with a Polhemus 3 Space Fasttrack (Polhemus, Colchester, Vermont). Five index points were determined to calculate the relative head position within the MEG helmet for source analysis. After MEG recordings, subjects rated each of the 75 affective pictures regarding emotional valence and arousal with the Self-Assessment Manikin (SAM) self-report scale.

\section{MEG Recording and Preprocessing}

The MEG was recorded continuously with a digitization rate of $678.17 \mathrm{~Hz}$ with a 148-channel whole head magnetometer (MAGNES $2500 \mathrm{WH}$, 4D Neuroimage, San Diego, California). A band-pass filter of $1-200 \mathrm{~Hz}$ was applied online. For artifact control, electrooculogram and electrocardiogram were recorded with a SynAmps amplifier (Neuroscan) with silver/silver chloride electrodes. Offline, global external noise and cardiac artifacts were corrected by means of procedures included in the MEG acquisition software package (Whole Head system software, version 1.2 .5 ; $4 \mathrm{D}$ Neuroimaging). Eye artifacts were corrected with the algorithm implemented in BESA software (24). The MEG data were digitally filtered between 1-Hz high-pass ( $6 \mathrm{~dB} /$ octave) and $25-\mathrm{Hz}$ low-pass (24 dB/octave). After artifact correction, trials containing amplitudes above $3.5 \mathrm{p} T$ (e.g., due to movement artifacts) were discarded from further analysis. The three groups did not differ in the number of accepted trials [Unexposed Group: mean $=73.9, \mathrm{SD}=2.3$, Trauma Control subjects: mean $=73.7, \mathrm{SD}$ $=2.2$, PTSD: mean $=72.5, \mathrm{SD}=6.7 ; F(2,70)=.61, p=.55]$. Finally, MEG data were averaged for picture category (pleasant, neutral, and aversive) over $1000 \mathrm{msec}$ (500-msec baseline and $500 \mathrm{msec}$ of stimulus presentation time).

\section{Source Analysis}

With the Matlab-based software EMEGS (25), the distribution of the cortical sources of neuromagnetic activity was estimated by calculating L2-minimum-norm solutions that offer enhanced resolution of brain activity generated by a magnetic field without a priori assumption regarding the location and number of current sources (26; Supplement 1). Calculation of the L2-minimumnorm was based on a one-shell spherical head model with 2 (azimuth and polar direction) $\times 197$ evenly distributed dipolar sources. A shell radius of $6 \mathrm{~cm}$ was chosen as the best tradeoff 
Table 1. Demographic and Clinical Characteristic of the Three Participant Groups

\begin{tabular}{|c|c|c|c|c|}
\hline & & $\begin{array}{c}\text { PTSD } \\
(n=36)\end{array}$ & $\begin{array}{l}\text { Trauma Control Subjects } \\
\qquad(n=21)\end{array}$ & $\begin{array}{l}\text { Unexposed } \\
\quad(n=16)\end{array}$ \\
\hline \multicolumn{5}{|l|}{ Demographic Data } \\
\hline \multicolumn{5}{|l|}{ Sex } \\
\hline Female & $n(\%)$ & $15(41.7)$ & $13(57.1)$ & $9(56.3)$ \\
\hline Male & $n(\%)$ & $21(58.3)$ & $9(42.9)$ & $7(43.8)$ \\
\hline Age & $M(S D)$ & $33.6(10.0)^{a}$ & $31.4(11.2)^{a}$ & $26.0(6.6)^{b}$ \\
\hline \multicolumn{5}{|l|}{ Regions of Origin } \\
\hline Middle East & $n(\%)$ & $19(52.8)^{a}$ & $8(38.1)^{b}$ & $6(37.5)^{b}$ \\
\hline The Balkans & $n(\%)$ & $3(8.3)$ & $6(28.6)$ & $9(56.3)$ \\
\hline Caucasus & $n(\%)$ & $2(5.6)$ & 0 & 0 \\
\hline Asia & $n(\%)$ & $3(8.3)$ & $3(14.3)$ & 0 \\
\hline Africa & $n(\%)$ & $9(25.0)$ & $4(19.1)$ & $1(6.3)$ \\
\hline \multicolumn{5}{|l|}{ Clinical Data } \\
\hline \multicolumn{5}{|l|}{ Events } \\
\hline No. Traumatic Events & & & & \\
\hline CAPS Event-Types & $M(S D)$ & $7.0(2.1)^{a}$ & $5.6(1.6)^{b}$ & $1.6(1.0)^{c}$ \\
\hline \multicolumn{5}{|l|}{ Vivo Checklist } \\
\hline No. war \& torture-types & $M(S D)$ & $10.9(5.8)^{a}$ & $4.1(5.4)^{b}$ & $.5(1.1)^{c}$ \\
\hline \multicolumn{5}{|l|}{ Clinical Symptoms } \\
\hline CAPS score & $M(S D)$ & $80.5(16.6)^{a}$ & $13.6(17.4)^{b}$ & $.6(2.3)^{c}$ \\
\hline Intrusions score & $M(S D)$ & $26.6(7.2)^{a}$ & $5.0(7.1)^{b}$ & $.1(.3)^{c}$ \\
\hline Avoidance score & $M(S D)$ & $28.4(8.2)^{a}$ & $4.8(6.4)^{b}$ & $.3(1.3)^{c}$ \\
\hline Arousal score & $M(S D)$ & $25.4(5.9)^{a}$ & $3.8(5.2)^{b}$ & $.3(1.0)^{c}$ \\
\hline \multicolumn{5}{|c|}{ Comorbid Disorders (MINI Diagnoses) } \\
\hline MD current & $n(\%)$ & $27(75)^{a}$ & $2(9.5)^{b}$ & $0^{b}$ \\
\hline Dysthymia & $n(\%)$ & $3(8.3)$ & $2(9.5)$ & 0 \\
\hline HDRS score & $M(S D)$ & $25.6(7.7)^{a}$ & $7.3(7.6)^{b}$ & $1.4(2.2)^{c}$ \\
\hline \multicolumn{5}{|l|}{ Medication } \\
\hline Antidepressants & $n(\%)$ & $8(22.2)$ & $2(9.5)$ & 0 \\
\hline Hypnotics & $n(\%)$ & $3(8.3)$ & $1(4.8)$ & 0 \\
\hline Neuroleptics & $n(\%)$ & $2(5.6)$ & $2(9.5)$ & 0 \\
\hline Anxiolytics & $n(\%)$ & $2(5.6)$ & 0 & 0 \\
\hline
\end{tabular}

For pair-wise group comparisons of continuous variables, Mann-Whitney $U$ Tests were used; differences of categorical variables were evaluated by applying $\chi^{2}$ tests for independence. Different indices $(a, b, \eta)$ indicate statistical difference $(p<.05)$ between the reported results.

CAPS, Clinical Administered PTSD Scale; MINI, Mini International Neuropsychiatric Interview; MD, major depression; HDRS, Hamilton Depression Rating Scale; PTSD, posttraumatic stress disorder.

between depth sensitivity and spatial resolution (27). Before calculating the L2-minimum-norm estimate, sensor data were baseline-corrected (500 msec before stimulus).

Estimated dipole strength was averaged for each group separately. Because the aim of this analysis was to investigate temporally early picture processing and to avoid contamination by the gradually developing steady-stated response triggered by the flickering stimulation, analyses were limited to the first 300 msec after stimulus onset.

Time windows and regions of interests (ROIs) for the statistical analyses were determined in two steps. At first, time windows were selected on the basis of the time course of the global field power of the minimum norm, which gives the overall neural activity across all dipoles (Figure 1). We identified time windows with a minimum duration of $30 \mathrm{msec}$ that showed an apparent group difference of the emotion modulation. Four time windows were determined around the four obvious activity peaks (130-160 msec, 170-205 msec, 206-256 msec, and $257-$ $300 \mathrm{msec}$ after stimulus onset) (Table 2).

Secondly, for each group and each time window, condition differences were projected onto the surface of a three-dimensional model of a standard brain. Spatial ROIs were selected for each time window on the basis of a visual inspection of valence-dependent activation differences within the three groups of participants. Because the main hypothesis was related to the response toward aversive stimuli, we focused on the aversiveneutral contrast for this screening. Pleasant stimuli served to establish the valence but not arousal specificity of expected cortical effects. Because visual inspection suggested hemispheric differences, a factor Hemisphere was included in the statistical analysis. To avoid false positives, only regions where the spatial extent of the aversive-neutral contrast comprised at least eight adjacent dipoles were selected for further analysis. Figure 2 provides a schematic layout of the dipole groups used for statistical analyses.

\section{Statistical Analysis}

The SAM pleasure and arousal ratings were analyzed with repeated-measures analysis of variance (ANOVA) with Group (Unexposed, Trauma Control subjects, PTSD) as the between factor and Category (pleasant, neural, aversive) as the within factor (Table 2). Activity at ROIs was analyzed with repeatedmeasures ANOVAs with Group as the between factor and with Category as well as Hemisphere (left, right) as the within factors. Because our main interests centered on group-specific differences in affective picture processing, only significant Group $\times$ 


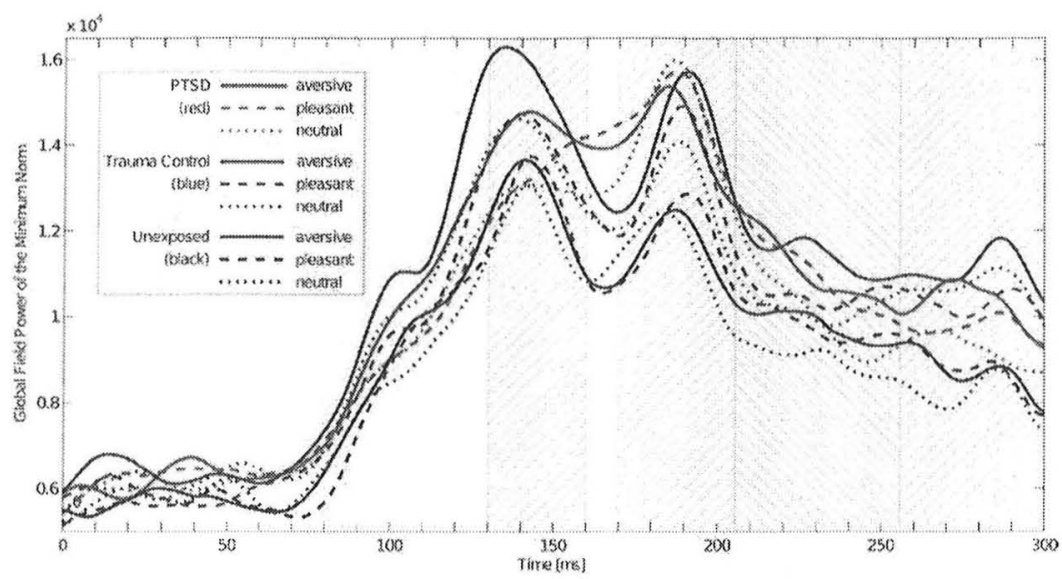

Figure 1. Global Field power of the magnetic field obtained at 197 dipoles for pleasant, neutral, and aversive pictures of each group separately. Shaded regions depict time windows of interests determined from the component structure of the data. PTSD, posttraumatic stress disorder.

Category $\times$ Hemisphere interactions within the ROIs were further analyzed. For a further exploration of the interaction effects, repeated measures ANOVAs were calculated for each group separately. Greenhouse-Geisser's corrections of the degrees of freedom were used where appropriate, and the associated epsilon and adjusted $p$ values are reported. Statistically significant interactions were further investigated by means of post hoc comparisons with the Tukey's Honestly Significant Difference test.

In addition, ANCOVAs were conducted with the HDSR score as covariate to explore the influence of depression on the results. Further exploratory correlative (Spearman rank) analyses were carried out to investigate the relationships between clinical variables and cortical activation patterns. The unexposed participants, who scored consistently low on all clinical instruments, were excluded from these analyses.

\section{Results}

\section{Ratings of Affective Stimuli}

The SAM arousal ratings differed significantly between picture categories $[F(2,106)=175.71, p<.001, \varepsilon=.88]$. Aversive pictures were rated as more arousing than neutral and pleasant pictures, and pleasant pictures were rated as more arousing than

Table 2. Regions of Interest

\begin{tabular}{|c|c|c|}
\hline Regions of Interests & Number of Dipoles & Group $\times$ Category $\times$ Hemisphere \\
\hline \multicolumn{3}{|l|}{$130-160$} \\
\hline Prefrontal & Total of 16 & $F(4,140)=2.47, p=.048$ \\
\hline Left & $60,61,82-84,108,109,132$ & \\
\hline Right & $78,79,103-105,127,128,150$ & \\
\hline Parietal & $\begin{array}{l}\text { Total of } 6 \\
14-16,28-30\end{array}$ & $F(4,140)=1.04, p=.389$ \\
\hline Occipital & Total of 18 & $F(4,140)=.41, p=.799$ \\
\hline Left & $45-47,67-69,90-92$ & \\
\hline Right & $48-50,70-72,95-97$ & \\
\hline \multicolumn{3}{|l|}{$170-205$} \\
\hline Prefrontal & Total of 20 & $F(4,140)=1.31, p=.271$ \\
\hline Left & $38,39,60,61,83,84,108,109,132,133$ & \\
\hline Right & $56,57,78,79,103,104,127,128,149,150$ & \\
\hline Occipital & Total of 22 & $F(4,140)=.92, p=.452$ \\
\hline Left & $26,27,44-46,66-68,90-92$ & \\
\hline Right & $30,31,49-51,72-73,95-97$ & \\
\hline \multicolumn{3}{|l|}{$206-256$} \\
\hline Prefrontal & Total of 20 & $F(4,140)=.93, p=.447$ \\
\hline Left & $38-41,59-62,82,83$ & \\
\hline Right & $54-57,77-80,104,105$ & \\
\hline Occipital & Total of 22 & $F(4,140)=2.50, p=.046$ \\
\hline Left & $27,28,45-47,67-69,91-93$ & \\
\hline Right & $29,30,48-50,70-72,94-96$ & \\
\hline \multicolumn{3}{|l|}{$256-300$} \\
\hline Prefrontal & Total of 20 & $F(4,140)=.40, p=.811$ \\
\hline Left & $38-41,59-62,82,83$ & \\
\hline Right & $54-57,77-80,104,105$ & \\
\hline Occipital & Total of 22 & $F(4,140)=.63, p=.646$ \\
\hline Left & $27,28,45-47,67-69,91-93$ & \\
\hline Right & $29,30,48-50,70-72,94-96$ & \\
\hline
\end{tabular}




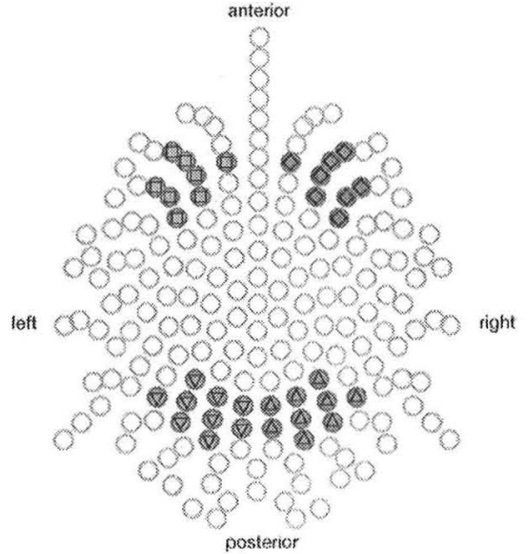

Dipole groups used for sfatisfical analysis ( $130-160 \mathrm{~ms}$ )

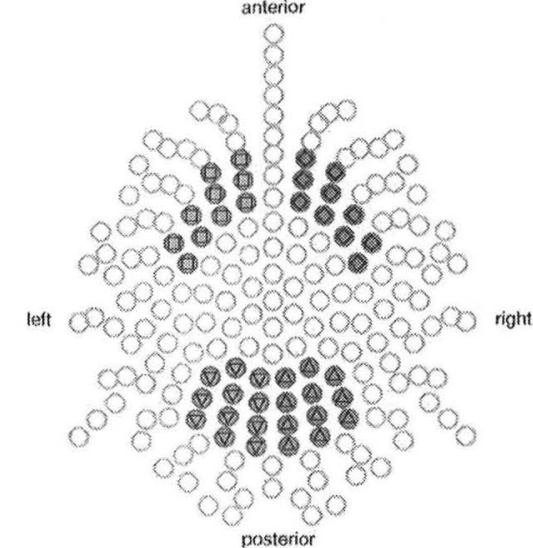

Dipole groups used for stafistical analysis $(206-256 \mathrm{~ms})$.
Figure 2. Schematic layout of the dipole distribution used for the L2 minimum norm estimation. Dipole groups used for the statistical analysis are marked. neutral pictures (all comparisons $p<.001$ ). Moreover, arousal ratings revealed a significant interaction effect of group $\times$ category $[F(4,106)=5.48, p<.001, \varepsilon=.88]$, suggesting that PTSD patients rated pleasant pictures as less arousing and unpleasant pictures as more arousing than the Unexposed group. However, these post hoc tests did not reach significance. Analyses of the SAM valence ratings showed a significant main effect of Group $[F(2,53)=9.34, p<.001]$. The PTSD patients rated all pictures as less pleasant than the other groups (Tukey's Honestly Significant Difference $p<.001$ for the comparison PTSD vs. Trauma Control subjects and $p<.05$ for Unexposed vs. P'TSD) (Figure 3). After the MEG recordings, some participants were fatigued and had difficulties concentrating on the ratings of the pictures. Therefore, only 14 Unexposed, 16 Trauma Control subjects, and 26 P'TSD participants completed SAM ratings.

\section{Minimum Norm Estimates}

Although main effects of picture category were present in all selected time windows, significant Group $\times$ Condition $\times$ Hemisphere interactions were only found within the time windows of 130-160 msec and 206-256 msec.

For the identified ROI in the ventral prefrontal cortex at $130-160 \mathrm{msec}$ this interaction reached $F(4,140)=2.47, p<.05$. Individual ANOVAs for each group revealed a significant Cate-

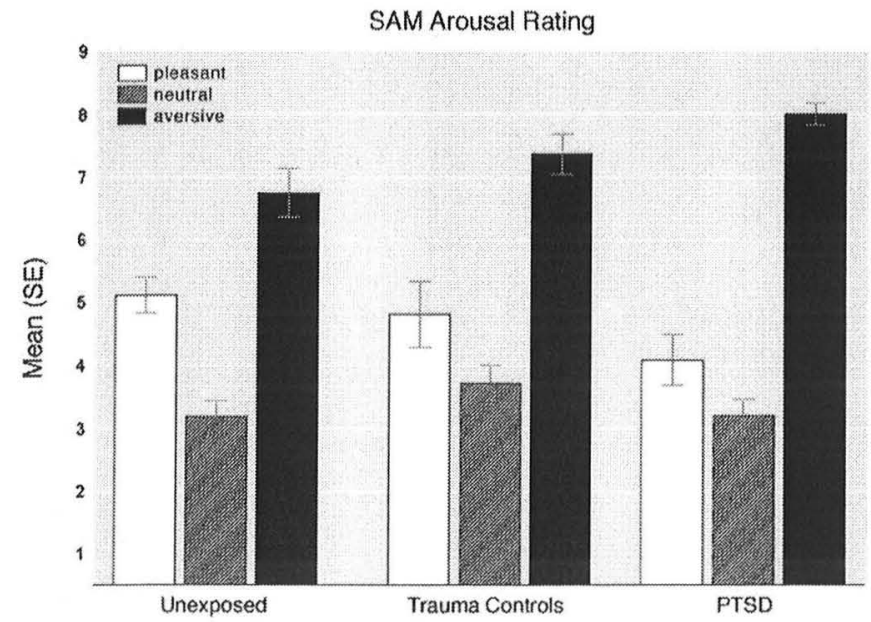

gory effect only in the PTSD group for the right hemisphere $[F(2,70)=8.43, p<.001]$. The PTSD patients showed significantly enhanced dipole source strength toward aversive pictures, compared with neutral, as well as toward pleasant pictures (Tukey's $p<.005$ for both comparisons). There was no significant effect of Category in either of the two control groups (Figure 4). Within the time period of $206-256 \mathrm{msec}$, a significant interaction of Group $\times$ Category $\times$ Hemisphere $[F(4,140)=$ $2.50, p<.05$ ] was found in the parieto-occipital ROI. Here, individual ANOVAs revealed a significant effect of Category in the right hemisphere only in the Trauma Control subjects $[F(2,40)=$ $14.36, p<.001]$ and in the Unexposed group $[F(2,30)=7.38, p<$ $.005]$. Both groups showed significantly higher dipole source strength toward emotional pictures than toward pictures of neutral content (Trauma Control subjects: Tukey's $p<.001$ for aversive vs. neutral and $p<.05$ for pleasant vs. neutral; Unexposed Group: $p<.05$ for aversive vs. neutral and $p<.005$ for pleasant vs. neutral). By contrast, PTSD patients did not show any significant arousal modulation at this processing stage (Figure 4). After including the HDSR score as covariate, the Group $x$ Category $\times$ Hemisphere remained significant only on a statistical trend level $(p=.08$ for the occipital effect, and $p=.15$ for the frontal effect). In the correlative analyses, we found a significant correlation of the aversive-neutral condition difference in the

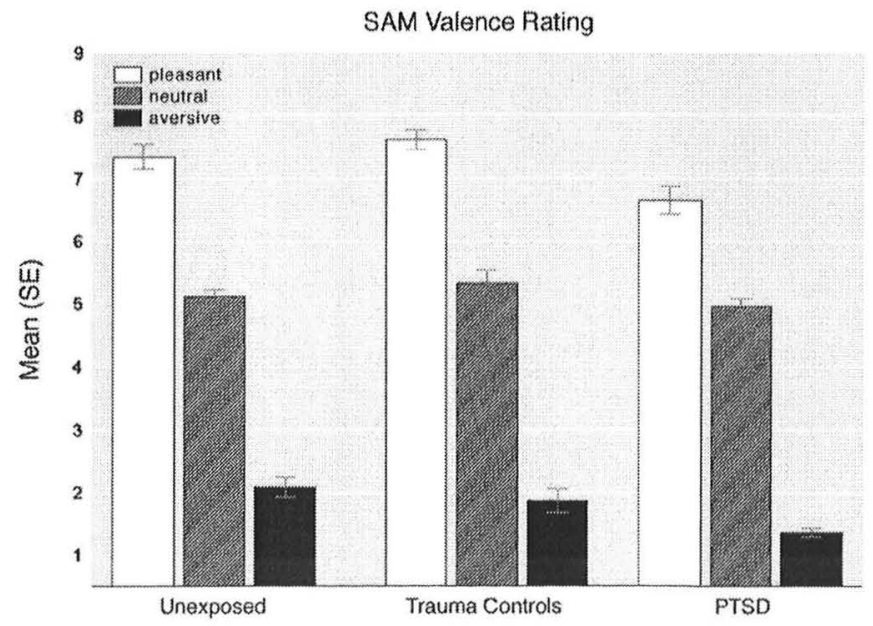

Figure 3. Means of Self-Assessment Manikin (SAM) arousal (left) and SAM valence (right) ratings of the three groups. Error bars indicate standard errors. 

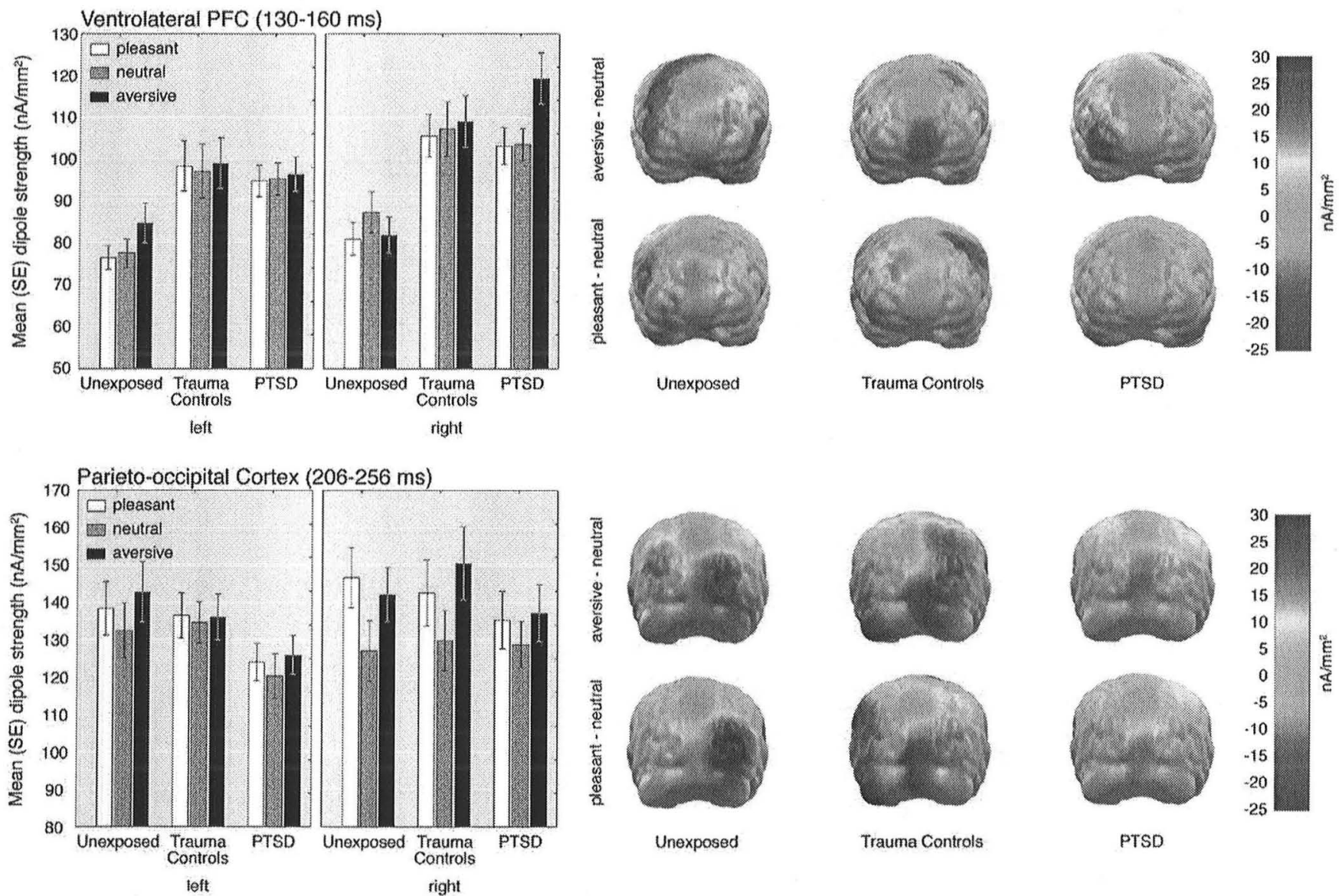

Unexposed

Trauma Controls

PTSD

Figure 4. Brain maps showing source activity (in $\mathrm{nAm}^{2}$ ) of the condition contrasts (aversive-neutral and pleasant-neutral) for the three groups (right). Repeated measures analysis of variance depict the statistical differences in the cortical activity within the selected regions of interest (left). PFC, prefrontal cortex; PTSD, posttraumatic stress disorder.

posterior ROI with the CAPS $(r=-.27, p<.05)$ but not with the HRSD $(p=.97)$, which indicates that this effect is associated with PTSD but not depression. The initial anterior hyperactivation and the subsequent posterior hypoactivation were not significantly correlated.

\section{Discussion}

As predicted, we found evidence for a biphasic cortical reaction in patients with PTSD compared with trauma-exposed and non-trauma-exposed healthy control subjects within the first $300 \mathrm{msec}$ of visual processing. This response consists of an augmentation of reactivity toward aversive stimuli in prefrontal areas followed by a decrease of the affective modulation in the parieto-occipital cortex. This finding is consistent with the hypothesis of a vigilant-avoidant reaction pattern to threat cues in anxiety disorders.

As early as 130-160 msec after stimulus onset, PTSD subjects showed an exaggerated prefrontal neural response to emotional stimuli (Figure 4). Notably, this effect was specific for aversive stimuli. This result confirms a previous study by Junghöfer $e t$ al. (18), who found a similar early response to aversive pictures in PTSD in orbitofrontal regions. Recent research has shown that the ventral prefrontal cortex has an active role in object recognition (28) and that neurons in this region provide a rapid affective categorization of visual stimuli within this time window
(6). These findings are consistent with the idea of a rapid threat detection mechanism that occurs independently of a detailed visual stimulus analysis (5) and involves not only subcortical brain structures but also prefrontal cortical areas $(4,29,30)$.

The prefrontal hyperactivation to aversive stimuli found in the present neuromagnetic imaging study, however, seems to contradict hemodynamic brain imaging research with PTSD patients that generally demonstrate the opposite effect (i.e., a prefrontal hypoactivation rather than hyperactivation in response to aversive stimuli) (8). An explanation for the diverse findings of MEG and functional magnetic resonance imaging (fMRI) research could be that the overresponsiveness found in the signals was transient and generally lasted no longer than $30-80 \mathrm{msec}$. Such a process can readily be missed in $\mathrm{FRR}$, given the low temporal resolution. Some authors, however, have suggested that it is possible to trace rapid transient neural processes with the fMRI by presenting stimuli subliminally. Following this logic, a recent fMRI study employed a nonconscious presentation of affective faces to investigate rapid brain responses in PTSD (31). The authors found, in contrast to the findings with overt presentation and in agreement with our study, an increased prefrontal activity after affective stimulation in PTSD patients. The early prefrontal activity might have been caused by an excitatory bottom-up influence of the amygdala on the prefrontal cortex $(29,32)$, which is consistent with a rapid initiation of a widespread neuronal fear network. 
Consistent with our hypothesis, we found that the prefrontal hyperactivation in PTSD was followed by an attenuated activity toward high-arousing stimuli in the parieto-occipital cortex in a later time window $(206-256 \mathrm{msec})$. Our findings confirm the results of recent ERP studies that also found reduced posterior ERP signals in response to affective faces (10) or affective pictures (12) in patients with trauma-related psychopathology. Our results, however, are in contrast to other ERP studies that found increased event-related electrical activation in PTSD compared with healthy control subjects in posterior sites of the brain in response to threatening visual stimuli $(33,34)$. A likely explanation for the discrepant findings might be that the posterior reaction to aversive stimuli depends on stimulus intensity and perceived self-relatedness. In two previous studies, for example, P'TSD participants showed a continuously decreasing brain response to tones as they were presented with an increasing intensity $(35,36)$. It could be speculated that PTSD subjects respond with enhanced brain activity toward aversive stimulation up to a certain threshold. Beyond this threshold, neural processes reverse as they instigate a state of protective inhibition of cortical activity to protect the cortex from overstimulation (37). Even though the pictures used in our study were not deliberately chosen to be trauma-related, many of the pictures might have triggered memories related to the individual traumatic experiences in our sample and thus exceeded the level of general aversive stimulation.

Although the early hyperactivation was specific for aversive stimuli, the later disengagement involved negative as well as positive stimuli. This finding is in line with the other studies that found a reduced cortical response toward arousing stimuli in traumatized subjects $(14,15)$. It seems that the neuronal avoidant reaction includes all types of arousing stimuli, which corresponds to the nonspecific emotional numbness in PTSD subjects.

The so-called hypervigilance-avoidance pattern found in the present study is characterized by an initial attentional vigilance followed by an attentional avoidance, which is a finding that has already been demonstrated for anxious participants in a recent ERP study by Holmes et al. (38). The biphasic process allows a rapid detection of threat cues that initiates an immediate flight or fight response. It might therefore reflect an adaptation of the cortical processing in individuals who live in a threatening or traumatizing environment. Once the stimulus has been classified as dangerous, further and more detailed analyses might not be necessary, thus leading to a reduced attention allocation. The analysis of the sustained cortical response observed in this experiment revealed that the avoidant reaction remained for at least $4 \mathrm{sec}(11)$, in agreement with this supposition.

Our findings can be interpreted as attention effects, on the basis of the motivated attention theory (39). Because we did not measure any behavioral data, however, it is premature to assume that the cortical processes revealed in this study underlie the behavioral vigilance-avoidance effect $(14,40,41)$. Further investigations should employ paradigms that allow studying behavioral reactions and cortical processes simultaneously to relate these phenomena.

Except for the association of the severity of PTSD and the cortical avoidance, the correlative analyses did not reveal significant effects. This does not necessarily argue against our findings, because the group differences might be based on nonlinear relationships.

The present study chose a sample of severely traumatized subjects (victims of war and torture) to study the correlates of trauma-related psychopathology. Although the intense trauma history as well as the high level of psychopathology increased the probability of finding neuronal correlates, several characteristics of the sample impair the unequivocal interpretation of the results. Because there were substantial between-group differences in comorbid disorders, particularly with respect to depression, our results reflect the correlates of trauma-related psychopathology rather than solely PTSD. Because recent studies $(42,43)$ have found a reduced arousal modulation in posterior regions, it is plausible to argue that comorbid depression might have contributed to the posterior disengagement effect in our study. However, including depression as a covariate did not seem to eliminate the effects completely, which indicates that the group differences might not be solely attributed to depression. Unfortunately, the design of our study does not allow separating the influence of PTSD from the effect of depression (44). This problem, however, is inherent in most PTSD research. Current research implies that the phenomenology of depression and PTSD overlaps considerably (45), and it has been argued that symptoms of depression and PTSD might be expressions of a unitary traumatic stress reaction (46). Therefore, it is questionable whether the differentiation of PTSD and depression is useful in this context at all.

Moreover, by stimulating with flickering pictures, we used an unusual mode of presentation. Although this stimulation allows the identification of sustained effects by analyzing the steadystate signal (11) as well as the immediate effects based on the ERF signal, it is not clear how the presentation affects the early potentials in comparison with a constant stimulation. Because we restricted the ERF analysis on the first $300 \mathrm{msec}$, however, there is no reason to assume that a stimulation frequency of $10 \mathrm{~Hz}$ would have dramatic effects on the data.

The various methods in neuroscience have different possibilities and limitations, in particular with respect to the temporal and spatial resolution of the observations. It seems that the detection of the cortical vigilance-avoidance pattern in this study depended on the high temporal resolution of the MEG measurement. Although current source localization methods allow a fairly precise localization of the likely spots of activity, the MEG does not allow studying subcortical processes. However, various subcortical regions, including the amygdala, have a key function in the fear network and seem to be related to the attentional bias toward threat $(47,48)$, which could not be identified in this study. Only the synopsis of the results from various methods will allow formulating comprehensive models of fear reactions.

Taken together, our findings contribute to the integration of seemingly contradictory findings in the assessment of alterations of brain responses to threatening stimuli in PTSD. The commonly found opposing results of over- and under-responsiveness might reflect the correlates of at least two different processes that are temporally and spatially dissociated.

The Deutsche Forschungsgemeinschaft and the European Refugee Fund supported the research. The authors report no biomedical financial interests or potential conflicts of interest. The corresponding author had full access to all of the data in the study and takes responsibility for the integrity of the data and the accuracy of the data analysis.

We are grateful to all participants who took part in the study. We thank the outpatient clinic staff for clinical support, Heike Riedke for logistical support, and Ursula Lommen for belping with MEG data acquisition. Moreover, we thank Anne Hauswald for technical support, and Katy Robjant for editing.

ClinicalTrials.gov: Change of Neural Network Indicators 
Through Narrative Treatment of PTSD in Torture Victims; bttp:// www.clinicaltrials.gov/ct2/show; NCT00563888. online.

Supplementary material cited in this article is available

1. Ehlers A, Clark DM (2000): A cognitive model of posttraumatic stress disorder. Behav Res Ther 38:319-345.

2. McNally R, Clancy S, Schacter D, Pitman R (2000): Cognitive processing of trauma cues in adults reporting repressed, recovered, or continuous memories of childhood sexual abuse. J Abnorm Psych 109:355.

3. Buckley TC, Blanchard EB, Neill WT (2000): Information processing and PTSD: A review of the empirical literature. Clin Psych Review 20:10411065.

4. Liddell BJ, Brown KJ, Kemp AH, Barton MJ, Das P, Peduto A, et al. (2005): A direct brainstem-amygdala-cortical "alarm" system for subliminal signals of fear. Neuroimage 24:235-243.

5. LeDoux JE (2000): Emotion circuits in the brain. Annu Rev Neurosci 23: 155-184.

6. Kawasaki H, Kaufman O, Damasio H, Damasio AR, Granner M, Bakken H, et al. (2001): Single-neuron responses to emotional visual stimuli recorded in human ventral prefrontal cortex. Nat Neurosci 4:15-16.

7. Bryant RA, Kemp AH, Felmingham KL, Liddell B, Olivieri G, Peduto A, et al. (2008): Enhanced amygdala and medial prefrontal activation during nonconscious processing of fear in posttraumatic stress disorder: An fMRI study. Hum Brain Mapp 29:517-523.

8. Shin LM, Rauch SL, Pitman RK (2006): Amygdala, medial prefrontal cortex, and hippocampal function in PTSD. Ann N Y Acad Sci 1071:67-79.

9. Karl A, Malta LS, Maercker A (2006): Meta-analytic review of eventrelated potential studies in post-traumatic stress disorder. Biol Psych 71:123-147.

10. Felmingham KL, Bryant RA, Gordon E (2003): Processing angry and neutral faces in post-traumatic stress disorder: An event-related potentials study. Neuroreport 14:777.

11. Catani C, Adenauer H, Keil J, Aichinger H, Neuner F (2009): Pattern of cortical activation during processing of aversive stimuli in traumatized survivors of war and torture. Eur Arch Psychiatry Clin Neurosci 259:340351.

12. Weber K, Miller GA, Schupp HT, Borgelt J, Awiszus B, Popov T, et al. (2009): Early life stress and psychiatric disorder modulate cortical responses to affective stimuli. Psychophysiology 46:1234-1243.

13. Koster EHW, Verschuere B, Crombez G, Van Damme S (2005): Timecourse of attention for threatening pictures in high and low trait anxiety. Behav Res Ther 43:1087-1098.

14. Pflugshaupt T, Mosimann U, Wartburg R, Schmitt W, Nyffeler T, Müri RM (2005): Hypervigilance avoidance pattern in spider phobia. J Anxiety Disord 19:105-116.

15. Mogg K, Bradley BP, Miles F, Dixon R (2004): Time course of attentional bias for threat scenes: Testing the vigilance-avoidance hypothesis. Cogn Emotion 18:689-700.

16. Junghoefer M, Sabatinelli D, Bradley MM, Schupp HT, Elbert TR, Lang PJ (2006): Fleeting images: Rapid affect discrimination in the visual cortex. Neuroreport 17:225.

17. Schupp HT, Flaisch T, Stockburger J, Junghoefer M (2006): Emotion and attention: Event-related brain potential studies. Prog Brain Res 156:31-51.

18. Junghöfer $M$, Schauer M, Neuner F, Odenwald M, Rockstroh B, Elbert T (2003): Enhanced fear-network in torture survivors activated by RVSP of aversive material can be monitored by magnetic source imaging. Psychophysiology 40:51.

19. Blake DD, Weathers FW, Nagy LM, Kaloupek DG, Gusman FD, Charney DS, Keane TM (1995): The development of a Clinician-Administered PTSD Scale. J Trauma Stress 8:75-90.

20. Vivo (2006): Vivo checklist of war, detention and torture events. Available at: http://www.vivofoundation.net. Accessed May 14, 2010.

21. Sheehan DV, Lecrubier $Y$, Sheehan $K H$, Amorim P, Janavs J, Weiller $E$, et al. (1998): The mini-international neuropsychiatric interview (M.I.N.I.): The development and validation of a structured diagnostic psychiatric interview for DSM-IV and ICD-10. J Clin Psychiatry 59(suppl 20):22-33; quiz:34-57.

22. Williams JB (1988): A structured interview guide for the Hamilton Depression Rating Scale. Arch Gen Psychiatry 45:742-747.
23. Lang PJ, Bradley MM, Cuthbert BN (2005): International affective picture system (IAPS): Affective ratings of pictures and instruction manual. Gainesville, Florida: University of Florida.

24. Berg P, Scherg M (1994): A multiple source approach to the correction of eye artifacts. Electroencephalogr Clin Neurophysiol 90:229-241.

25. Junghöfer M, Peyk P (2004): Analysis of electrical potentials and magnetic fields of the brain. Matlab Select. 2:24-28 EMEGS software. Available at: http://www.emegs.org. Accessed May 14, 2010.

26. Hämäläinen M, Ilmoniemi R (1994): Interpreting magnetic fields of the brain: Minimum norm estimates. Med Biol Eng Comput 32:35-42.

27. Hauk $O$ (2004): Keep it simple: A case for using classical minimum norm estimation in the analysis of EEG and MEG data. Neuroimage 21:1612-1621.

28. Bar M, Kassam K, Ghuman A, Boshyan J, Schmid A, Dale A, et al. (2006): Top-down Facilitation of Visual Recognition. Proc Natl Acad Sci U S A 103:449.

29. Liddell BJ, Williams LM, Rathjen J, Shevrin H, Gordon E (2004): A temporal dissociation of subliminal versus supraliminal fear perception: An event-related potential study. J Cognit Neurosci 16:479-486.

30. Williams LM, Das P, Liddell BJ, Kemp AH, Rennie CJ, Gordon E (2006): Mode of functional connectivity in amygdala pathways dissociates level of awareness for signals of fear. J Neurosci 26:9264.

31. Bryant RA, Kemp AH, Felmingham KL, Liddell B, Olivieri G, Peduto A, et al. (2008): Enhanced amygdala and medial prefrontal activation during nonconscious processing of fear in posttraumatic stress disorder: An fMRI study. Hum Brain Mapp 29:517-23.

32. Gilboa A, Shalev AY, Laor L, Lester H, Louzoun Y, Chisin R, Bonne O (2004): Functional connectivity of the prefrontal cortex and the amygdala in posttraumatic stress disorder. Biol Psychiatry 55:263-272.

33. Attias J, Bleich A, Furman V, Zinger $Y$ (1996): Event-related potentials in post-traumatic stress disorder of combat origin. Biol Psychiatry 40:373-381.

34. Bleich A, Attias J, Furman V (1996): Effect of repeated visual traumatic stimuli on the event related $\mathrm{P} 3$ brain potential in post-traumatic stress disorder. Int J Neurosci 85:45-55.

35. Paige SR, Reid GM, Allen MG, Newton JE (1990): Psychophysiological correlates of posttraumatic stress disorder in Vietnam veterans. Biol Psychiatry 27:419.

36. Lewine JD, Thoma RJ, Provencal SL, Edgar C, Miller GA, Canive JM (2002): Abnormal stimulus-response intensity functions in posttraumatic stress disorder: An electrophysiological investigation. Am J Psychiatry 159: 1689-1695.

37. Buchsbaum M, Pfefferbaum A (1971): Individual differences in stimulus intensity response. Psychophysiology 8:600-611.

38. Holmes A, Nielsen MK, Green S (2008): Effects of anxiety on the processing of fearful and happy faces: An event-related potential study. Biol Psychol 77:159-173.

39. Lang PJ, Bradley MM, Cuthbert BN (1997): Motivated attention: Affect, activation, and action. In: Lang PJ, Simons RF, Balaban MT, editors. Attention and Orienting: Sensory and Motivational Processes. Hillsdale, New Jersey: Lawrence Erlbaum Associates, 97-134.

40. Garner M, Mogg K, Bradley B (2006): Orienting and maintenance of gaze to facial expressions in social anxiety. J Abnorm Psych 115:760.

41. Mogg K, Garner M, Bradley BP (2007): Anxiety and orienting of gaze to angry and fearful faces. Biol Psych 76:163-169.

42. Moratti S, Rubio G, Campo P, Keil A, Ortiz T (2008): Hypofunction of right temporoparietal cortex during emotional arousal in depression. Arch Gen Psychiatry 65:532-541.

43. Kayser J, Bruder G, Tenke C, Stewart J, Quitkin F (2000): Event-related potentials (ERPs) to hemifield presentations of emotional stimuli: Differences between depressed patients and healthy adults in P3 amplitude and asymmetry. Int $J$ Psychophysiol 36:211-236.

44. Miller GA, Chapman JP (2001): Misunderstanding analysis of covariance. J Abnorm Psych 110:40-48.

45. Rosen G, Lilienfeld S (2008): Posttraumatic stress disorder: An empirical evaluation of core assumptions. Clin Psych Review 28:837-868.

46. O'Donnell M, Creamer M, Pattison P (2004): Posttraumatic stress disorder and depression following trauma:Understanding comorbidity. Am J Psychiatry 161:1390.

47. Monk C, Telzer E, Mogg K, Bradley B, Mai X, Louro H, et al. (2008): Amygdala and ventrolateral prefrontal cortex activation to masked angry faces in children and adolescents with generalized anxiety disorder. Arch Gen Psychiatry 65:568

48. Anderson A, Phelps E (2001): Lesions of the human amygdala impair enhanced perception of emotionally salient events. Nature 411:305-308. 